\section{Papers from an international symposium on quantitative genetics and plant breeding (Baton Rouge, Louisiana, March 2001)}

\author{
Quantitative Genetics, Genomics and Plant \\ Breeding \\ MS Kang \\ CABI Publishing, Oxford; 2002. 432 pp. \\ $£ 75.00$, hardback. ISBN 0-85199-601-9
}

Heredity (2003) 91, 94. doi:10.1038/sj.hdy.6800319

\section{Reviewed by JE Flintham}

The wide range of subjects touched upon in this volume has resulted in necessarily brief treatments in parts (the main exceptions are some detailed accounts of statistical methods used for genotype/environment interactions). As such, it is most likely to appeal to the interested student of biometry seeking a general introduction to a variety of relevant disciplines - a book to borrow from the library, rather than a 'must buy' text. Although the field has continued to progress exponentially since the contributions were originally compiled, the take-home messages remain valid and demand serious attention. One overarching requirement that emerges is the urgent need for crosstalk and synthesis between different disciplines, relevant to researchers and breeders concerned with crop improvement.

The first (and most entertaining) chapter is a collection of historical vignettes, illustrating some of the potential pitfalls to be encountered in the field of quantitative genetics. I felt particular sympathy with the reporter of a meeting, who did not understand the 'apparently beautiful' papers in statistical genetics, but who hoped that their authors did so. The main body of the book is in two sections; chapters $2-14$ deal largely with biotechnological and bioinformatic tools for quantitative genetics in plants, while techniques for analysing and modelling genotype/environment interactions are discussed in chapters 15-24.

A case is made for the importance of quantitative genetics for exploiting large-scale genomics resources, with a brief review of potential plant breeding applications for techniques from animal breeding, evolutionary and human genetics. An overview of bioinformatics introduces a compelling argument for systematic integration of quantitative trait locus (QTL), genomic, and transcriptome analyses. Answering central questions in quantitative genetics will require fine-mapping of QTL candidate genes; technical difficulties in achieving this goal are explained, and the production of stepwise aligned recombinant lines is proposed as a solution. An alternative approach, association mapping, can exploit pre-existent germplasm (cultivars, landraces, breeding lines) although the precision attainable is not yet clear for most crops. The potential benefits of integrating markerassisted selection (MAS), transformation, and genomics into quantitative genetics and breeding are assessed and compared with the power of conventional, variancebased analyses. In either case, it is concluded that applications to traits controlled by more than about ten genes are unlikely to prove cost effective in the short to medium term.

Overviews of current (2001) status for QTL analyses and applications in breeding for components of grain yield and quality, plant development, disease and drought resistance are given for maize, barley and rice, together with a theoretical evaluation of the efficiency of MAS for introgressing useful traits via backcrossing. Computer simulations of phenotypic outputs from complex genetic networks, and crop responses to environmental cues, raise questions as to how far predictive biology may be able to respond to the challenge of complexity in crop productivity. A short chapter on tissue culture techniques for crop improvement links to the other topics via its importance in clonal propagation, doubled-haploid, and transformation protocols. Section I concludes with a review of successful introgressions of improved traits from wild relatives into rice.

Section II opens with an examination of the criticality of genotype/environment interaction (GE) in determining crop performance, and its consequences for genetic analyses and selection. Brief mention is made of the potential for participatory breeding, involving more local expertise and germplasm, both in meeting the needs of developing agriculture and in broadening the genetic bases of breeding programmes. Various chapters detail statistical theory behind graphical biplot analyses for genotype/trait, diallel, and genotype/pathotype data, linear/bilinear and mixed GE models. Practical examples are presented covering a broad range of species and environments, for traits including yield, nitrogen use efficiency, drought and photoperiod responses. The final chapter compares different approaches to improving crop stability and adaptation, including an analytical flowchart for planning a selection strategy. These chapters are a useful compendium for anyone analysing experiments replicated over genotypes, times and locations.

For plant breeders, farmers and consumers, proof of the 'biotech pudding' will literally be in the eating. This book includes some sober appraisals of the current ability of massive investments, in DNA marker development, genome mapping and sequencing, bioinformatics, transcriptome and computational analyses, to deliver real benefits in terms of world food security. The need to translate outstanding achievements from laboratory to plate is clear and present; much remains to be done in bridging between the two. As a closing comment, the following quote may be worthy of wider consideration (JW Dudley, p. 80): 'Without public research investment in plant breeding and quantitative genetics training, the potential benefits of genomics research will not be realized.' 